\title{
RBF Neural Network Controller Research Based on AFSA Algorithm
}

\author{
Qing-kun Song, Meng-meng Xu and Yi Liu \\ School of Automation \\ Harbin University of Science And Technology \\ Control theory and control engineering \\ sqk@hrbust.edu.cn,xumm_1988@163.com,459033664@qq.com
}

\begin{abstract}
Artificial fish-swarm algorithm is a realization model of the swarm intelligence optimization algorithm. It uses the optimization model of imitated nature fish for feeding from top to bottom, clusters and rear, local optimization by individuat fish, achleve the purpose of global optimal values highlighted in the groups. RBFNN based on the AFSA can accurately find the optimal solution quickly and ensure the diversity of artificial fish. It is easier to find the global optimal point of optimal fish. This design uses second-order pendulum as a controlled object, using artificial fish swarm algorthm applied to the neural network training algorithms, building design of RBF Neural networks control module, verifing by Matlab simulation of actual control controller performance.
\end{abstract}

Keywords: RBF neural network: Artificial fish swarm algorithm; Double inverted pendulum

\section{Introduction}

Artificial neural networks [1] are nonlinear system of massively parallel distributed processing, application of neural network theory has penetrated into all areas. Artificial fishswarm algorithm is an organic earch optimization algorithm based on simulation of fish behavior, it makes use of fish feeding, clusters and rear, from the structural behavior of a single fish up, by individual fish local optimization purposes of global optimization. Algorithm has the ability to overcome the local extreme values, access to global extremism. This article for double inverted pendulum control model, the introduction of artificial fishswarm algorithm n optimization of radial basis function neural network training, the establishment of a training model of neural network controller based on the algorithm and has a better performance simulation controller.

\section{The Basic Theory:}

RBP neural network topology shown in Figure 1. RBF network transformation is nonlinear from the input space to the hidden space, whereas is linear from the hidden layer space to the output space. This is a feed-forward network topology. 


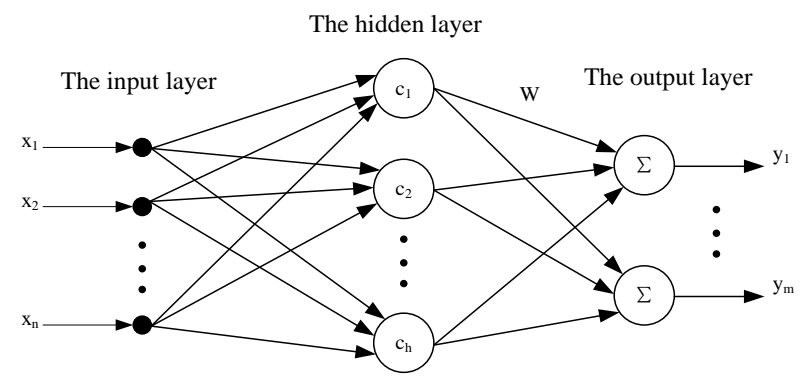

Figure 1. The Structure of RBF Neural Network

Network topology affect greatly the performance of the neural network, there are four main factors decided of the RBF network topology: RBF center [2] vector, the number of hidden nodes, the width of the radial basis function, and the weights of matrix from hidden layer to the output layer. Gaussian function [3] is often used as the commonly used activation function in radial basis function can be expressed as:

$$
\Phi_{j}(x)=\exp \left(-\frac{\left\|x-c_{j}\right\|^{2}}{2 \sigma_{j}^{2}}\right.
$$

Where $\delta_{\mathrm{j}}$ is Gaussian function of variance. $\| \mathrm{x}-\mathrm{c}_{\mathrm{j}} \mathrm{l}$ is European norm, $\mathrm{C}_{\mathrm{j}}$ said that the center of the Gaussian function[4]. The $\delta_{j}$ smaller, the smaller the width of the radial basis function, and basis function will hold the stronger selective

Then the k-th output of the RBF networkgan be expressed as:

$$
\left.\mathrm{y}_{\mathrm{y}}=\sum_{j=1}^{n} w_{k j} \Phi_{j}()_{0}\right)
$$

$w_{k j}$ is weights value between layer neurons and output layer neurons, $\mathrm{y}_{\mathrm{k}}$ is actual output. RBF neural network learnifg methods of the learning process in two phases: First determine the center of all hidden layer nodes $\mathrm{C}_{\mathrm{j}}$ and radius, then correct the connection weights between the hidden layer and batput layer matrix in the $\mathrm{W}$. The training method of the algorithm steps we can see from the reference article [5].

\section{RBF Neural Networ Artificial Fish Swarm Algorithm:}

Artificial fish swarm algorithm which based on research in intelligent behavior of animal groups is a new bonic optimization algorithm LI Xiao-lei [6] in 2002. Upon this algorithm, in this paper get an algorithm which is RBF Neural network artificial fish swarm algorithm.

This algorithm is mainly used to optimize the RBFNN [7] in hidden layer node position and width value, this artificial fish need to determine the encoding and initialed, calculated to determine the fitness value of artificial fish behavior, calculate the hidden layer to the output laye yeights to determine RBFNN output error, the paper is divided into steps to address these issues.

Step 1: The initialized first step, the location of the initial value of the hidden units training samples, assuming that the training sample matrix in Figure 2 shows. 


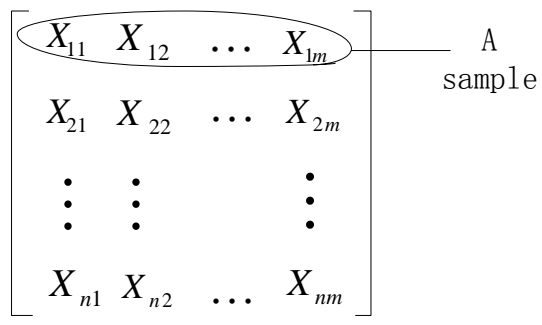

Figure 2. Initialize the Training Sample Matrix

Where, $\mathrm{m}$ is the number of the dimension of the input samples, that is, the number of neuronsin the input layer. The initialization $n$ is the number of training samples. The initialization set mimin is the $\mathrm{i}$-th column of the minimum value in the training sample, the initialization is the difference of the maximum and minimum values of the $i$-th colunn, $M$ is a constant. Then the minimum value of the $\mathrm{i}$-th column may be expressed as:

$$
\operatorname{hid}_{j i}=X_{i \mathrm{~min}}+\operatorname{rand}(0, M),(\Delta>M)
$$

Step 2: Selecting the range of the width values can be defined within a relatively large range of search. Setting the upper range $\delta_{\max }$ is initialize all central value the maximum Euclidean enough distance twice. The lower range $\delta_{\min }$ is the lower limit is to initialize all the center value of the minimum Euclidean distance of 0.5 times. $\Delta i=\delta_{\max }-\delta_{\min }$. The value of the width of the i-th hidden unit can be expressedas:

$$
\sigma_{\wedge}=\sigma_{\min }+\operatorname{rand}(0, M) \cdot(\Delta \sigma / M)
$$

Through the two-step initialization of the center value of the hidden layer nodes to make the particle swarm [8] is more evenly distributed throughout the solution space.

RBF neural network parameters performance in order to easily, we select each artificial fish represents a feasible solution to the reciprocal of the mean square error as the fitness value [9], which i-artificial fish fitness value can be expressed as:

$$
\text { ? } f(i)=1 / E(i)=1 / \sum_{k=i}^{N} \sum_{j=1}^{M}\left|d_{k j}-y_{k j}\right|
$$

Where $\mathrm{N}$ represents the number of input samples, $\mathrm{M}$ represents the number of the output node.

The location of each artificial fish represents a feasible solution to the RBF neural network. Therefore, each artificial fish should include the hidden units, width, as well as the fitness value. There is $m$ hidden units and $n$-dim hidden unit. $M * n+m+1$-dimensional data representation for each artificial fish are available. The individual fish coding structure is shown in Figure 3:

$$
C_{11} C_{12} \ldots C_{1 \mathrm{n}} C_{21} C_{22} \ldots C_{2 n} \ldots \ldots C_{m 1} C_{m 2} \ldots C_{m n} \sigma_{1} \sigma_{2} \ldots \sigma_{m} f_{i}
$$

\section{Figure 3. The Individual Fish Coding Structure}

Where $C_{i j}$ is the first n-dimensional data of the m-th hidden unit.

Using the Fig.2 encoding allows the algorithm to continue to seek more appropriate in the process of search for a global minimum point due to unit location, and to ensure that each hidden unit corresponds to the width value. 


\section{Experimental Results and Simulation}

The object of this paper is the double inverted pendulum, Figure 4 shows the two inverted pendulum system diagram. In the figure 4 , the next cosmetic and angle is $\theta_{1}$, Swing speed under is $\dot{\theta}_{1}$, On cosmetic and angle is $\theta_{2}$, On Swing speed is $\dot{\theta}_{2}$, The displacement of the trolley is $x$, The speed of the trolley is $\dot{x}$, Inverted pendulum system input to the force applied on the trolley is $F$. The six inverted pendulum system state variables are expressed as:

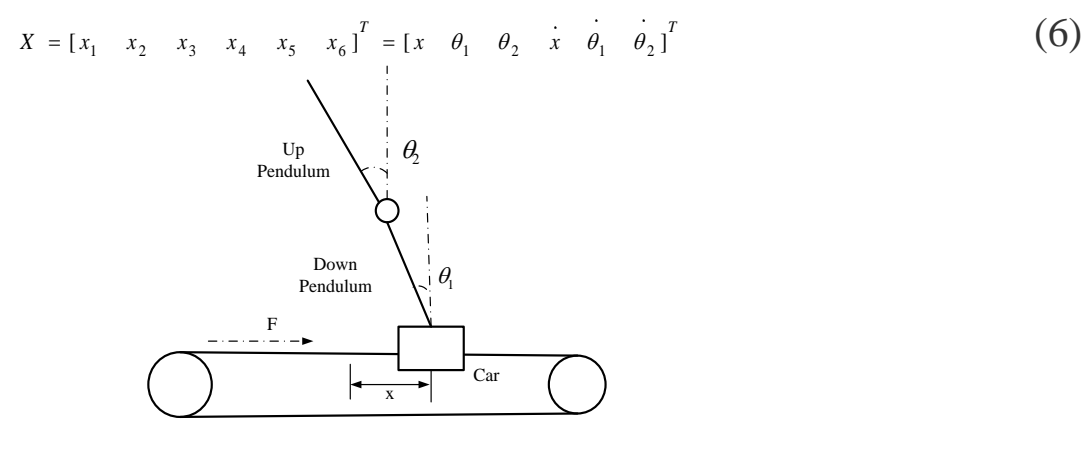

Figure 4. The Structure Diagram of Double Invertéd Pendulum

Parameters for double inverted pendulum: the quality of car $M=1.32 \mathrm{~kg}$, the quality of hem $m_{1}=0.04 \mathrm{~kg}$, On the pendulum mass $m_{2}=0.132 \mathrm{~kg}$, the quality of the mass on the pendulum and hem is $0.208 \mathrm{~kg}$, Hem lever rotation center to he rodand the distance of the centroid $l_{1}=0.09 \mathrm{~m}$, On the swing lever rotation center othe rod and the distance of the centroid $l_{2}=0.27 \mathrm{~m} . \mathrm{F}$ is the external force acting on the system, $F$ that $1 S$ the amount of control in the system state space $u$, and $u=F$.

The state space of the system described in the equations of motion can be derived from Lagrange:

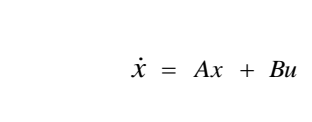

Into various physical parameters can be obtained, the value of the system matrix:

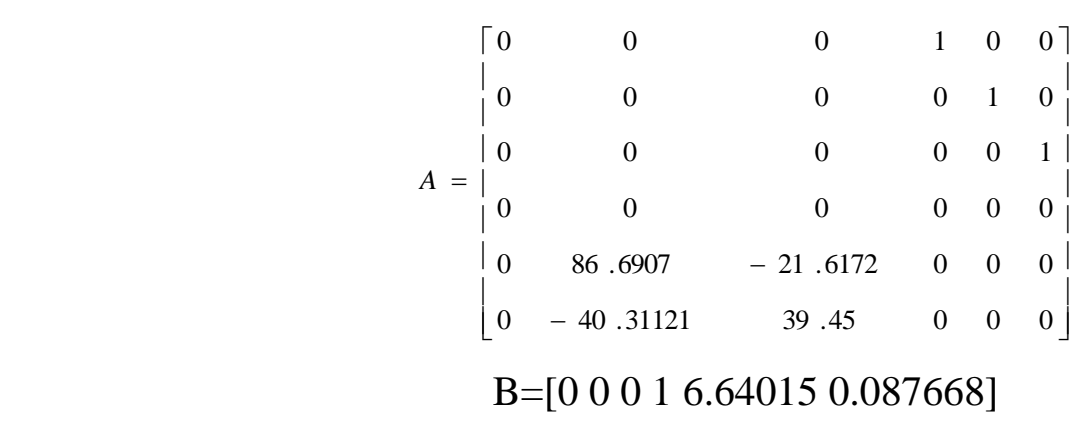

The populations number is 50 , the initial range is set to [-1,1], the sample data is 55 group. Network of hidden nodes is 6 . When the error of the network is no longer changes obviously, the application change to stop the iteration until the error reached 0.00005 . The results obtaining is shown in Figure 5. 


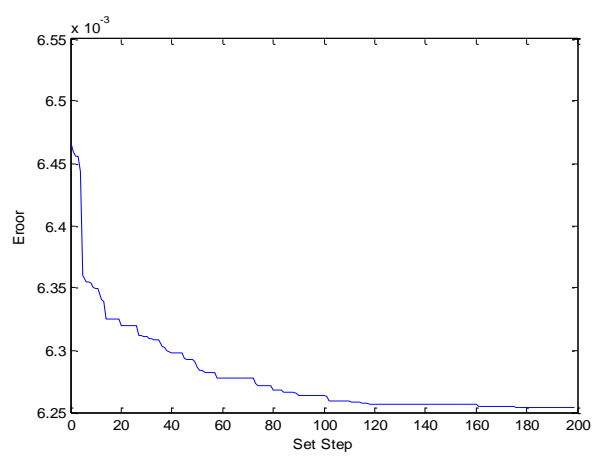

Figure 5. Figure of the Error Trend in the Training of SFSA

Data after 55 sets of data extracted through the use of optimization algorithms to optimize neural network controller to complete the fitting with the source data, proved the robustness of the controller and optimize the effect.

Artificial fish swarm algorithm applied directly to the RBF neural network, network error0.00005 no longer change after 200 steps to reach the enor requirement. This shows that the artificial fish swarm algorithm enables the RBF neural network initial value select appropriate, overcome the genetic algorithm to search a long time, the slow shortcomings, and a fast and reliable optimization of RBF neural network nethod. The results obtaining is shown in Figure 6.

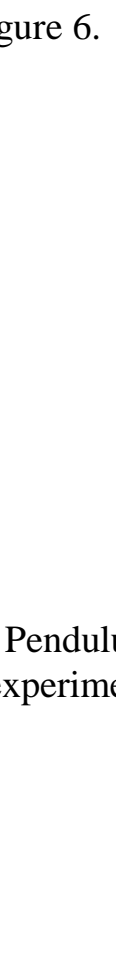

Inverted Pendulam RBF network controller based on artificial fish swarm algorithm simulation experiment, the results obtained is shown in Figure 7:
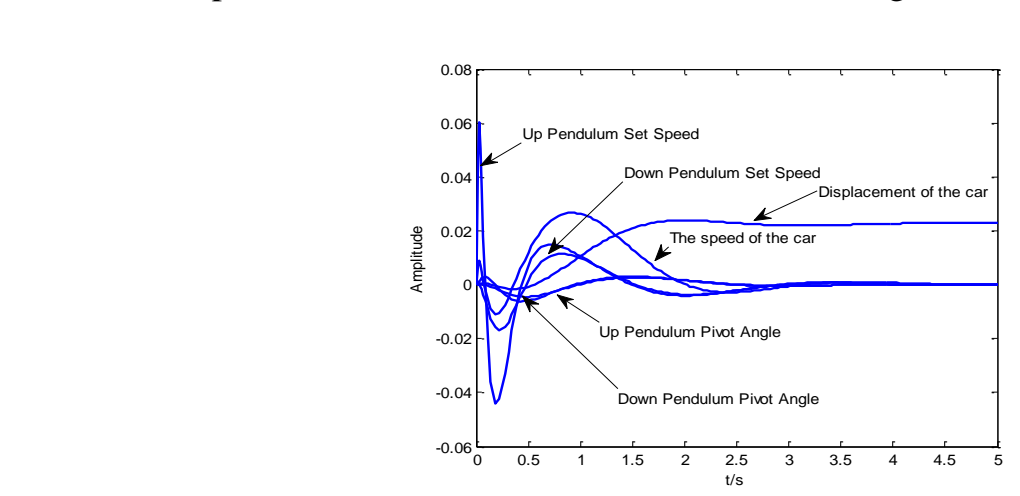

Figure 7. The RBF Control Simulation Curve of Double Inverted Pendulum 
Simulation results show that this controller can achieve stable the double inverted pendulum system to take the actual experiment sampling period and experimental of several interference to test the controller performance, the test results are shown in Figure 8:
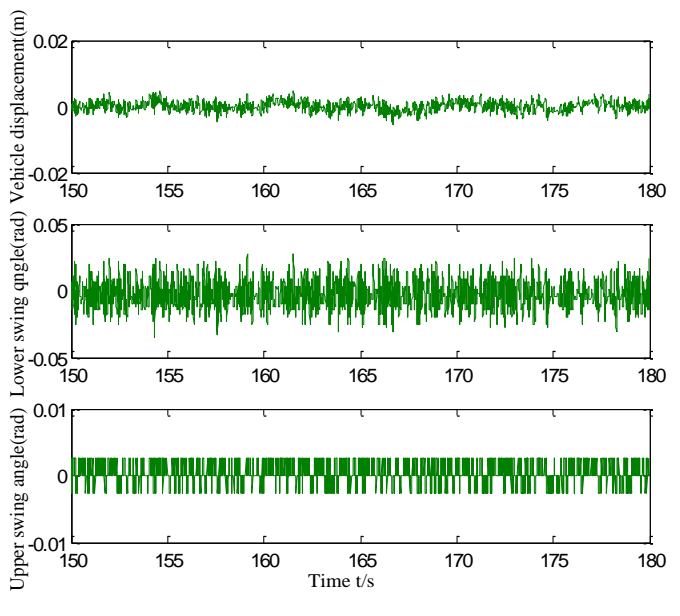

Figure 8. Figure of Inverted Pendulum System under $T=0.005 \mathrm{~s}$

\section{Conclusion}

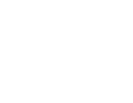

In this paper, RBF neural network is based on artficial fish swarm algorithm training model, forming a new RBF network traning algorithm. The method has good convergence, the initial values not sensitive and nnsensitive parameters characteristics; algorithm has a faster convergence rate algorithm convergence process has obvious advantages. Not only do how to better feeding, cluster and rear-end three optimization behavior, reflected in the handling oft he algorithm Diake computing easier, but also the need to further improve. Finally, the simulation results can be seen, the controller designed in this paper can be stable control of double inverted pendulum system, and has a good anti-jamming capability. From the actual control curve to be seen, the design of the RBF neural network controller based on artificial fish warmalgorithmis effective.

\section{References}

[1] F. Yan, "Artifician neural networks and evolutionary computing", 2nd edition, Tsinghua University Publishing House, (2005), pp. 101-105.

[2] M. J. L. Orr, "Intcoduction to Radial Basis Function networks", Scotland: University os Edinburgh, Center for Cognitive Science, (1996).

[3] H. Hong-ton "A New Bi-Directional Selection RBFNN Algorithm Based on Gaussian Function", Computer Science vol. 34, no. 7, (2007), pp. 211-213.

[4] S. Karim, "Online Identification of nonlinear multivariable processes using self-generating RBF neural networks", Asian Journal of Control, vol. 9, (2010), pp. 626-639.

[5] J. B. Gomm and D. LiYu, "Selecting radial basis function network centers with recursive orthogonal least 3quares training", vol. 02, (2000).

[6] L. Xiao-Lei, X. Yun-can and L. Fei, "Parameter Estimation Method Based-on Artificial school Algorithm", Journal of Shandong University, Engineering Science, vol. 34, no. 3, (2004), pp. 84-87.

[7] L. Yao-nian, L. Ying-hong and L. Jun-feng, "RBF neural Network Based on Artifical Fish Swarm Algorithm", Journal of Northeast Dianli University, Natural Science Edition, vol. 26, no. 4, (2006), pp. 2327.

[8] C. M. Bishop, "Improving the generalization properties of radial basis function neural networks", Neural Computation, vol. 3, (2010), pp. 579-581.

[9] H. Hong-ton, "A New Bi-Directional Selection RBFNN Algorithm Based on Gaussian Function", Computer Science, vol. 34, no. 7, (2007), pp. 211-213. 